\title{
Rapidly Expanding Infectious Aortic Aneurysm Caused by Perforated Colon Cancer
}

\author{
Taufiek Konrad Rajab, MD, BChir ${ }^{1}$, Daniel E. Rinewalt, MD ${ }^{1}$, Michael Belkin, MD', \\ Joel E. Goldberg, MD', Haiyang Zhou, MD* \\ 1 Brigham and Women's Hospital and Harvard Medical School, Boston, Massachusetts, USA \\ ${ }^{2}$ Changzheng Hospital and Second Military Medical University, Shanghai, People's Republic of China
}

\begin{abstract}
A 50-year-old male smoker presented with a perforated colon cancer and underwent an extended right colectomy. Feculent peritonitis was treated with empiric antibiotics. Postoperatively he developed severe back pain and rising leukocytosis. Serial computed tomography revealed a rapidly expanding infrarenal aortic aneurysm. He was urgently treated with extra-anatomic bypasses and aortic resection. No organisms grew from the resected aortic wall. He was discharged in stable condition, and the ileostomy was reversed 9 months later.

Copyright $\odot 2016$ Science International Corp.
\end{abstract}

\section{Key Words}

Infected aortic aneurysm - Colon cancer - Aorta • Inflammation • Aneurysm

\section{Introduction}

The etymology of aneurysm derives from the Greek

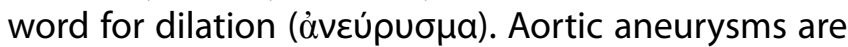
full-thickness dilations of the aorta that exceed its normal diameter by 50\% [1]. The role of infectious micro-organisms in the development of aortic aneurysms was classically described by Sir William Osler in 1885 [2]. With the advent of antibiotics, infectious aortic aneurysms have become very rare $[3,4]$. Here we describe an infectious aortic aneurysm caused by perforated colon cancer.

\section{Case Presentation}

A 50-year-old male smoker presented with acute onset abdominal pain and peritoneal signs. Exploration revealed a perforated transverse colon mass that was treated with an extended right colectomy with mobilization of the splenic flexure and end ileostomy. Pathology of the mass showed medullary colon carcinoma with stage $\mathrm{T4b} \mathrm{N} 1 \mathrm{a}$ (1 out of 30 positive regional lymph nodes). Postoperatively the patient was started on empiric vancomycin and piperacillin/tazobactam to prevent feculent peritonitis. His postoperative course was complicated by delayed return of bowel function and persistent leukocytosis. Computed tomography (CT) on postoperative day 6 demonstrated evidence of partial bowel obstruction. The patient also complained of progressive back pain that was not controlled with opiates. A second opinion on the $C T$ scan was sought from a cardiovascular radiologist, who noted mild retroperitoneal stranding around the aorta (Figure 1). The patient made a good further recovery but continued to complain of back pain. Repeat CT scan on postoperative day 15 showed new aneurysmal dilation of the infrarenal aorta with a dramatic increase in diameterfrom $3.3 \mathrm{~cm}$ to $5.5 \mathrm{~cm}$ since the first CT scan 9 days earlier. Moreover, a saccular contour irregularity at the left posterior aspect of the aneurysm and worsened peri-aortic stranding were noted (Figure 2). These findings were suggestive of an infectious aortic aneurysm with concern for impending rupture, and the patient underwent emergent surgery.

\footnotetext{
* Corresponding Author:

Haiyang Zhou, MD

Department of Surgery

Changzheng Hospital, Second Military Medical University

415 Fengyang Road, Shanghai, 200003, China

Tel: +86 218188 5598; Fax: +86 218188 5591; E-Mail: haiyang1985_1@aliyun.com
} 


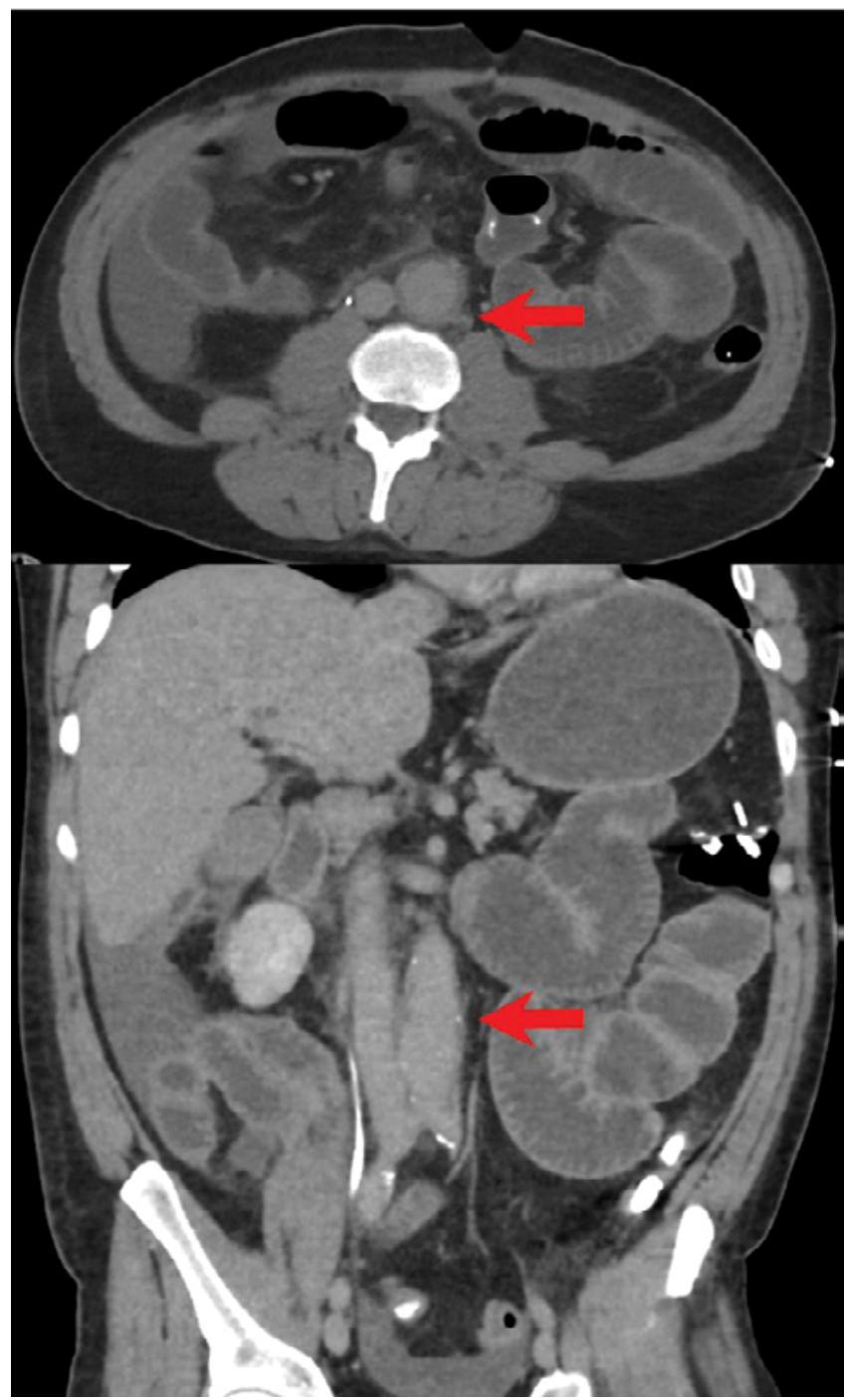

Figure 1. Computed tomography scan on postoperative day 6 in cross-sectional and coronal views showing mild stranding around the aorta (arrows).

In the first part of the operation, axillary-to-femoral and femoral-to-femoral polytetrafluoroethylene bypass grafts were constructed in a sterile field. The contaminatedabdominalfield wasaddressed in the second half of the surgery. The old laparotomy was re-entered, and dense adhesions were removed. Exposure of the infrarenal aorta revealed a prominent aortic aneurysm with surrounding necrotic and inflamed tissue but no frank purulence. Proximal and distal control were obtained by clamping the infrarenal aorta and common iliac arteries, respectively. The involved aortic segment and both proximal iliac arteries were resected. Bleeding lumbar arteries were oversewn with figure-of-eight polypropylene sutures.

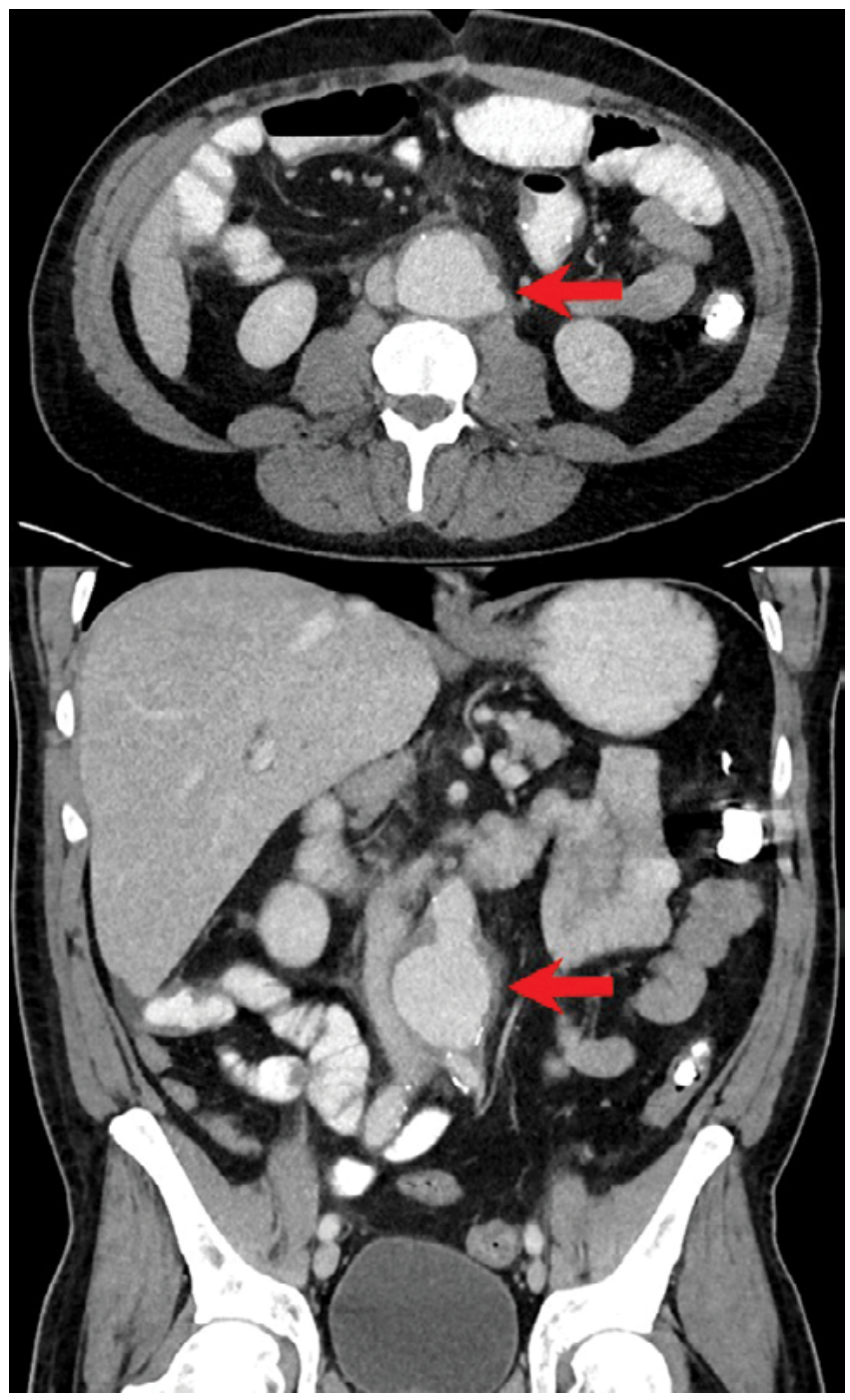

Figure 2. Computed tomography scan on postoperative day 15 in cross-sectional and coronal views showing aneurysmal dilation of the infrarenal aorta (arrows).

The aortic stump was closed with a polypropylene continuous horizontal mattress suture to obtain intimal apposition and a top layer polypropylene whip-stich to ensure hemostasis. The common iliac stumps were closed in the same fashion. The contaminated field was then debrided and irrigated by pulsed lavage with $3 \mathrm{~L}$ normal saline solution containing cefazolin, vancomycin, and gentamycin. The retroperitoneum was closed over the arterial stumps, but no omental pedicle flaps were available for additional coverage. Pathology of the resected aorta showed a polymorphonuclear infiltrate, but no organisms were seen, and culture results were also negative. The patient's postoperative course was 
unremarkable, and he was discharged on intravenous vancomycin and meropenem to a rehabilitation facility. His ileostomy was removed 9 months later, and he was well at the 1-year postoperative follow-up.

\section{Discussion}

Infectious aortic aneurysms feature amongst the most challenging problems in vascular surgery because they are rare, difficult to diagnose, difficult to manage, and rapidly fatal. Infectious aortic aneurysms constitute less than $1 \%$ of aortic aneurysms $[3,4]$. The diagnosis is based on history as well as clinical, laboratory, and radiographic manifestations of the infection and the aortic mass effect. In our patient, empiric antibiotics started at the time of colon cancer perforation masked the stigmata of infection and explain the negative culture results. Expeditious surgical management is critical because over half of infectious aortic aneurysms are already ruptured at the time of surgery $[5,6]$. In our patient, a saccular protuberance on CT was concerning for an impending rupture, so he was urgently taken for surgery. In such cases, extra-anatomic bypass grafting in a sterile field followed by aortic resection and retroperitoneal debridement achieve the surgical objectives by preventing aortic rupture, controlling sepsis, and reconstructing the vasculature.

This case highlights that a high index of suspicion with active investigation to determine the diagnosis and expeditious surgery are critical for successful management of infectious aortic aneurysms.

\section{Conflict of Interest}

The authors have no conflict of interest relevant to this publication.

\section{Comment on this Article or Ask a Question}

\section{References}

1. Johnston KW, Rutherford RB, Tilson MD, Shah DM, Hollier L, Stanley JC. Suggested standards for reporting on arterial aneurysms. Subcommittee on Reporting Standards for Arterial Aneurysms, Ad Hoc Committee on Reporting Standards, Society for Vascular Surgery and North American Chapter, International Society for Cardiovascular Surgery. J Vasc Surg. 1991;13:452-458. DOI: 10.1067/ mva.1991.26737

2. Osler W. The gulstonian lectures, on malignant endocarditis. BrMed J. 1885;1:577579. DOl: 10.1136/bmj.1.1264.577

3. Oderich GS, Panneton JM, Bower TC, Cherry KJ, Rowland CM, Noel AA, et al. Infected aortic aneurysms: aggressive presentation, complicated early outcome, but durable results. J Vasc Surg. 2001;34:900-908. DOI: 10.1067/ mva.2001.118084

4. Reddy DJ, Shepard AD, Evans JR, Wright DJ, Smith RF, Ernst CB. Management of infected aortoiliac aneurysms. Arch Surg. 1991;126:873-878. DOI: 10.1001/archsurg.1991.01410310083012

5. Chen IM, Chang HH, Hsu CP, Lai ST, Shih CC. Ten-year experience with surgical repair of mycotic aortic aneurysms. J Chin Med Assoc. 2005;68:265-271. DOI: 10.1016/S17264901(09)70148-0

6. Müller BT, Wegener OR, Grabitz K, Pillny M, Thomas L, Sandmann W. Mycotic aneurysms of the thoracic and abdominal aorta and iliac arteries: experience with anatomic and extra-anatomic repair in 33 cases. J Vasc Surg. 2001;33:106-113. DOI: 10.1067/mva.2001.110356
Cite this article as: Rajab TK, Rinewalt DE, Goldberg JE, Zhou H. Rapidly Expanding Infectious Aortic Aneurysm Caused by Perforated Colon Cancer. AORTA (Stamford). 2016;4(4):131-133. DOI: http://dx.doi.org/10.12945/j. aorta.2016.16.011 